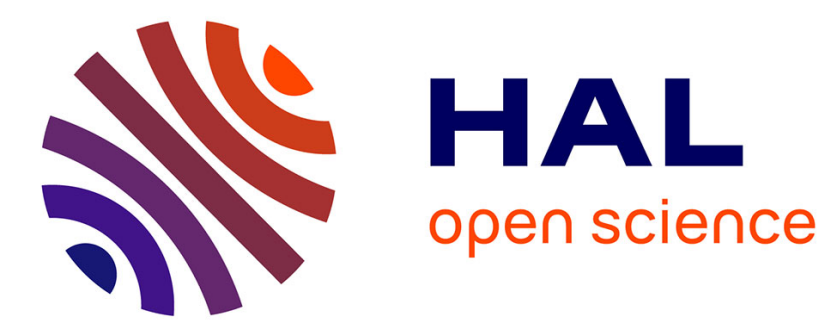

\title{
Lyapunov-based approach for finite time stability and stabilization
}

Emmanuel Moulay, W. Perruquetti

\section{To cite this version:}

Emmanuel Moulay, W. Perruquetti. Lyapunov-based approach for finite time stability and stabilization. 44th IEEE Conference on Decision and Control, 2005, Seville, Spain. pp.4742-4747, 10.1109/CDC.2005.1582911 . hal-03144982

\section{HAL Id: hal-03144982 \\ https://hal.science/hal-03144982}

Submitted on 18 Feb 2021

HAL is a multi-disciplinary open access archive for the deposit and dissemination of scientific research documents, whether they are published or not. The documents may come from teaching and research institutions in France or abroad, or from public or private research centers.
L'archive ouverte pluridisciplinaire $\mathbf{H A L}$, est destinée au dépôt et à la diffusion de documents scientifiques de niveau recherche, publiés ou non, émanant des établissements d'enseignement et de recherche français ou étrangers, des laboratoires publics ou privés. 


\title{
Lyapunov-based approach for finite time stability and stabilization
}

\author{
Emmanuel Moulay and Wilfrid Perruquetti
}

\begin{abstract}
Finite time stability is investigated for continuous system $\dot{x}=f(x)$ which satisfies uniqueness of solutions in forward time. A necessary and sufficient condition is given for this class of systems using Lyapunov functions. Then, a necessary and sufficient condition is developed for finite time stabilization of class $\mathcal{C} \mathcal{L}^{k}$-affine systems involving a class $\mathcal{C} \mathcal{L}^{0}$-settling time for the closed-loop system. Finally an explicit feedback control is given using a control Lyapunov function verifying a certain inequality.
\end{abstract}

\section{INTRODUCTION}

This paper deals with the finite time stability of systems with the uniqueness of solutions in forward time and the finite time stabilization problem of affine systems. The aim is to provide a necessary and sufficient condition for finite time stability and finite time stabilization. Lyapunovlike techniques have been successfully used to solve these problems. Finite time stability and stabilization have often been a subject of research. Thus, Haimo gives a sufficient condition for finite time stability of continuous systems

$$
\dot{x}=f(x), x \in \mathbb{R}^{n}
$$

in [8]. Bhat and Bernstein provided an important contribution in [4] by proving that there is a necessary and sufficient condition for finite time stability involving the continuity of the settling-time function at the origin. A part of our results is based on [4] (the reader can find some additional and useful results in this paper). In general (without the continuity of the settling-time function at the origin) such necessary and sufficient conditions have not been discussed in the literature.

The paper is organized as follows. After some notations in section II, section III states a general necessary and sufficient condition for the finite time stability using the properties of the settling-time function. Then a necessary and sufficient condition of finite time stabilization using the control Lyapunov function is addressed in section IV. Based on this result, an explicit feedback control is given under some less restrictive sufficient conditions.

\section{NOTATIONS}

Let us introduce some notations and definitions that will be useful later.

This work was supported in part by programs Maths-STIC CNRS 19 and ARCIR ROBOCOOP

E. Moulay is with Laboratoire Paul Painlevé, UFR de Mathématiques Pures et Appliquées, Université des Sciences et Technologies de Lille and LAGIS (UMR-CNRS 8021), Ecole centrale de Lille, Cité Scientifique, 59651 Villeneuve d'Ascq Cedex, France (e-mail: emmanuel.moulay@eclille.fr)

W. Perruquetti is with the LAGIS (UMR CNRS 8021), Ecole Centrale de Lille, (e-mail: wilfrid.perruquetti@ec-lille.fr)
Notation 1: Let $\mathcal{E}$ and $\mathcal{F}$ be two vector spaces and $k \geq 1$, we denote by $\mathcal{C} \mathcal{L}^{0}(\mathcal{E}, \mathcal{F})$ (respectively $\mathcal{C} \mathcal{L}^{k}(\mathcal{E}, \mathcal{F})$ ) the set of continuous functions on $\mathcal{E}$, locally Lipschitz on $\mathcal{E} \backslash\{0\}$ with value in $\mathcal{F}$ (respectively the set of continuous functions on $\mathcal{E}, C^{k}$ on $\mathcal{E} \backslash\{0\}$ with value in $\mathcal{F}$ ).

Throughout this paper, $\mathcal{V}$ will be a non empty neighborhood of the origin in $\mathbb{R}^{n}, \mathcal{B}^{n}$ the open unit ball in $\mathbb{R}^{n}$. As usually, a function $V: \mathcal{V} \rightarrow \mathbb{R}$ is proper if for every compact set $K \subset \mathbb{R}, V^{-1}(K)$ is compact. System (1) possesses unique solutions in forward time on $\mathcal{U} \subset \mathbb{R}^{n}$ if for all $x_{0} \in \mathcal{U}$ and two right maximally defined solutions of (1): $\phi^{x_{0}}:\left[0, T_{\phi}\left[\rightarrow \mathbb{R}^{n}\right.\right.$ and $\psi^{x_{0}}:\left[0, T_{\psi}\left[\rightarrow \mathbb{R}^{n}\right.\right.$, there exists $0<T_{x_{0}} \leq \min \left\{T_{\phi}, T_{\psi}\right\}$ such that $\phi^{x_{0}}(t)=\psi^{x_{0}}(t)$ for all $t \in\left[0, T_{x_{0}}\left[\right.\right.$. We may assume that for each $x_{0} \in \mathcal{U}, T_{x_{0}}$ is chosen to be the largest in $\overline{\mathbb{R}}_{\geq 0}$. In the following, $\phi^{x_{0}}(t)$ denotes a solution of system (1) starting from $x_{0} \in \mathbb{R}^{n}$ at $t=0$. Various sufficient conditions for forward uniqueness can be found in [1], [6, Chapter 10] or [10].

Now, let us recall some concepts of non-smooth analysis. Let $[a, b] \subset \mathbb{R}$, the upper Dini derivative of a function $f$ : $[a, b] \subset \mathbb{R} \rightarrow \mathbb{R}$ is the function $D^{+} f:[a, b] \rightarrow \overline{\mathbb{R}}$ defined by:

$$
D^{+} f(x)=\limsup _{h \rightarrow 0^{+}} \frac{f(x+h)-f(x)}{h} .
$$

If $V: \mathcal{V} \rightarrow \mathbb{R}$ is a continuous function, $\dot{V}$ is the upperright Dini derivative of $V$ along the solutions of (1), that is $\dot{V}(x)=D^{+}\left(V \circ \phi^{x}\right)(0)$. If $V$ is locally Lipschitz at $x \in \mathcal{V}$, then

$$
\dot{V}(x)=\limsup _{h \rightarrow 0^{+}} \frac{V(x+h f(x))-V(x)}{h}
$$

and if $V$ is continuously differentiable at $x \in \mathcal{V}$, then $\dot{V}(x)=\langle\nabla V(x), f(x)\rangle$. As it is customary in control theory, a Lyapunov function $V$ for the system (1) is a continuous positive definite function such that $\dot{V}$ is negative definite. The Lie derivative of $V: \mathbb{R}^{n} \rightarrow \mathbb{R}$ along $f: \mathbb{R}^{n} \rightarrow \mathbb{R}^{n}$ is defined by:

$$
\mathcal{L}_{f} V: \mathbb{R}^{n} \rightarrow \mathbb{R}, \quad \mathcal{L}_{f} V(x)=\langle\nabla V(x), f(x)\rangle .
$$

In section IV, we need some concept on set-valued functions. A set-valued function $\Phi$ from $\mathcal{X}$ to $\mathcal{Y}$ is a function that maps $x \in \mathcal{X}$ to a set $\Phi(x) \subset \mathcal{Y}$. Let $\mathcal{X}$ and $\mathcal{Y}$ be two vector spaces and $\Phi: \mathcal{X} \rightarrow \mathcal{Y}$ a set-valued function, $\Phi$ is lower semi-continuous if $\{x \in \mathcal{X}: \Phi(x) \cap O \neq \emptyset\}$ is open in $\mathcal{X}$ for every open set $O \subset \mathcal{Y}$.

$\Phi$ is locally Lipschitz if for any $x_{0} \in \mathcal{X}$, there exists a neighborhood $\mathcal{N}\left(x_{0}\right) \subset \mathcal{X}$ and a constant $l \geq 0$ such that for all $x, x^{\prime} \in \mathcal{N}\left(x_{0}\right)$,

$$
\Phi(x) \subset \Phi\left(x^{\prime}\right)+l\left\|x-x^{\prime}\right\|_{\mathcal{X}} \mathcal{B}^{\mathcal{Y}}
$$


where $\mathcal{B}^{\mathcal{Y}}$ is the unit ball in $\mathcal{Y}$.

\section{Finite TIME STABILITY OF SYSTEMS With THE UNIQUENESS OF SOLUTIONS IN FORWARD TIME}

Let us consider the system (1) where $f \in C^{0}\left(\mathbb{R}^{n}\right)$, $f(0)=0$ and when $f$ possesses the property of uniqueness of solutions in forward time. Let us recall the asymptotic stability which is a local property. The origin is asymptotically stable for the system (1) if:

1) the origin is stable for the system (1),

2) the origin of the system (1) is attractive: for all $\epsilon>0$, there exists $\delta(\epsilon)>0$ such that each solution starting from $x_{0} \in \delta(\epsilon) \mathcal{B}^{n}$ tends to the origin as $t$ tends to infinity.

Now, we may recall the notion of finite time stability involving the

settling-time function (see [4, Definition 2.2])

Definition 1: The origin is finite time stable for the system (1) if there exists a non empty neighborhood of the origin $\mathcal{V}$ in $\mathbb{R}^{n}$ such that:

1) there exists a function $T: \mathcal{V} \backslash\{0\} \rightarrow \mathbb{R}_{\geq 0}$ such that if $x_{0} \in \mathcal{V} \backslash\{0\}$ then $\phi^{x_{0}}(t)$ is defined (and particularly unique) on $\left[0, T\left(x_{0}\right)\left[, \phi^{x_{0}}(t) \in \mathcal{V} \backslash\{0\}\right.\right.$ for all $t \in$ $\left[0, T\left(x_{0}\right)\left[\right.\right.$ and $\lim _{t \rightarrow T\left(x_{0}\right)} \phi^{x_{0}}(t)=0 . T$ is called the settling-time of the system (1).

2) for all $\epsilon>0$, there exists $\delta(\epsilon)>0$, for every $x_{0} \in\left(\delta(\epsilon) \mathcal{B}^{n} \backslash\{0\}\right) \cap \mathcal{V}, \phi^{x_{0}}(t) \in \epsilon \mathcal{B}^{n}$ for all $t \in\left[0, T\left(x_{0}\right)[\right.$.

Remark 1: First, note that if the origin of system (1) is finite time stable, then $f$ cannot possess uniqueness in backward time at the origin, in particular $f$ cannot be locally Lipschitz at the origin.

Then, if system (1) is finite time stable, Lyapunov asymptotic stability implies that $\phi^{0} \equiv 0$ is the unique solution starting from $x_{0}=0$. So, the settling-time function $T$ may be extended at the origin by $T(0)=0$. We will also call this extension the settling-time of the system (1).

The following result is given in [4, Proposition 2.3].

Lemma 1: Suppose that the origin is finite time stable for the system (1) with the settling-time function $T: \mathcal{V} \rightarrow \mathbb{R}_{\geq 0}$, then for all $x \in \mathcal{V}$ the flow $\Phi(t, x)=\phi^{x}(t)$ of the system (1) is defined and continuous on $\mathbb{R}_{\geq 0} \times \mathcal{V}$ and $\Phi(t, x)=0$ for all $t \geq T(x)$.

This result shows that the finite time stability of system (1) implies:

- the uniqueness in forward time of solutions starting from $\mathcal{V}$

- the asymptotic stability,

- the existence of a continuous flow.

Finally, $T(x)$ is the time for the solution $\phi^{x}$ to reach the origin, and as the system is autonomous, the Lyapunov stability ensures that the solution stays at the origin for any time longer than $T(x)$. Then, the equality is as follows

$$
T(x)=\inf \left\{t \in \mathbb{R}_{\geq 0}: \Phi(t, x)=0\right\} .
$$

Let us recall the fundamental theorem of Kurzweil which is in [11, Theorem 7].

Theorem 1 (of Kurzweil): Let us consider the system (1) such that $f$ is continuous, the system (1) is asymptotically stable if and only if there exists a smooth Lyapunov function for the system (1).

Here, the theorem 1 of Kurzweil is of importance because we cannot have the Lipschitz continuity at the origin of the right-hand side of the system (1). Let us give the main result of this section which is a general necessary and sufficient condition for finite time stability.

Theorem 2: Let us consider the system (1) with uniqueness of solutions in forward time outside the origin, the following properties are equivalent:

(i) the origin of the system (1) is finite time stable on $\mathcal{V}$,

(ii) there exists a smooth Lyapunov function $V: \mathcal{V} \rightarrow \mathbb{R}_{\geq 0}$ for the system (1) satisfying for all $x \in \mathcal{V}$

$$
\int_{V(x)}^{0} \frac{d s}{\dot{V}\left(\Phi\left(\theta_{x}(s), x\right)\right)}<+\infty
$$

where $\theta_{x}$ is the inverse of $t \mapsto V(\Phi(t, x))$,

(iii) all smooth Lyapunov functions $V: \mathcal{V} \rightarrow \mathbb{R}_{\geq 0}$ for the system (1) satisfy for all $x \in \mathcal{V}$

$$
\int_{V(x)}^{0} \frac{d s}{\dot{V}\left(\Phi\left(\theta_{x}(s), x\right)\right)}<+\infty .
$$

Moreover, if $V$ is such a Lyapunov function then

$$
T(x)=\int_{V(x)}^{0} \frac{d s}{\dot{V}\left(\Phi\left(\theta_{x}(s), x\right)\right)} .
$$

Proof: $(i) \Rightarrow$ (ii) If the system (1) is finite time stable with the settling-time function $T: \mathcal{V} \rightarrow \mathbb{R}_{\geq 0}$, then, there exists a smooth Lyapunov function $V: \mathcal{V} \rightarrow \mathbb{R}^{n}$ for the system (1) given by the theorem 1 of Kurzweil. So, the well defined application $[0, T(x)[\rightarrow] 0, V(x)], t \mapsto$ $V(\Phi(t, x))$ is strictly decreasing and differentiable, so its inverse $] 0, V(x)] \rightarrow\left[0, T(x)\left[, s \mapsto \theta_{x}(s)\right.\right.$ is differentiable and satisfies for all $s \in] 0, V(x)]$,

$$
\theta_{x}^{\prime}(s)=\frac{1}{\dot{V}\left(\Phi\left(\theta_{x}(s), x\right)\right)} .
$$

The use of the change of variables $s=V(\Phi(t, x))$ leads to the following equalities

$$
\begin{aligned}
T(x) & =\int_{0}^{T(x)} d t=\int_{V(x)}^{0} \theta_{x}^{\prime}(s) d s \\
& =\int_{0}^{V(x)} \frac{d s}{-\dot{V}\left(\Phi\left(\theta_{x}(s), x\right)\right)}<+\infty .
\end{aligned}
$$

$($ ii $) \Rightarrow($ iii $)$ The set of smooth Lyapunov function for the system (1) $\mathbf{S}_{L}$ is non empty. Let $V \in \mathbf{S}_{L}$, then by using the same argument as before, we deduce that $V$ satisfies (3). ( iii $) \Rightarrow($ ( $)$ As there exists a Lyapunov function for the system (1), the theorem of Lyapunov (see [7]) ensures that it is asymptotically stable. The equalities (4) imply the finite time convergence. 
Remark 2: If the Lyapunov function $V$ is defined on $\mathbb{R}^{n}$, proper, and if the condition (ii) is globally held, then, the origin of the system (1) is globally finite time stable.

Even if theorem 2 is a theoretical result, we may give a simple example.

Example 1: Let us consider the Cauchy problem

$$
\left\{\begin{array}{l}
\dot{x}=-x \\
x(0)=1
\end{array}\right.
$$

and the smooth Lyapunov function $V(x)=\frac{x^{2}}{2}$. Then, $V(\phi(t))=e^{-2 t}, \theta(s)=-\frac{1}{2} \ln (s)$ and $\phi(\theta(s))=\sqrt{s}$ lead to $\dot{V}(\phi(\theta(s)))=-s$ where $s>0$. We have

$$
T(1)=\int_{0}^{\frac{1}{2}} \frac{d s}{s}=+\infty .
$$

Theorem (2) ensures that the system $\dot{x}=-x$ is not finite time stable.

In general, the settling-time function is not continuous at the origin. A fundamental example is given in [4, Example 2.2] which shows that the settling-time function of a finite time stable system is generally non continuous at the origin.

Now, we may give a corollary to theorem 2 which gives a sufficient condition for a continuous (or class $\mathcal{C L}^{0}$ ) settlingtime function.

Corollary 1: Let us consider the system (1) with the uniqueness of solutions in forward time outside the origin. Let us assume that there exists a smooth Lyapunov function $V: \mathcal{V} \rightarrow \mathbb{R}_{\geq 0}$ for the system (1) and $g \in$ $L^{1}\left(\left[0, \sup _{x \in \mathcal{V}} V(x)\right]\right)$ such that for all $x \in \mathcal{V} \backslash\{0\}$, and all $s \in[0, V(x)]$

$$
\frac{-1}{\dot{V}\left(\Phi\left(\theta_{x}(s), x\right)\right)} \leq g(s)
$$

then the system (1) is finite time stable with a continuous settling-time function.

Proof: If there exists a smooth Lyapunov function $V: \mathcal{V} \rightarrow \mathbb{R}_{\geq 0}$ for the system and a function $g \in L^{1}\left(\left[0, \sup _{x \in \mathcal{V}} V(x)\right]\right)$ such that for all $x \in \mathcal{V} \backslash$ $\{0\}$, and all $t \in[0, V(x)], \frac{-1}{\dot{V}\left(\Phi\left(\theta_{x}(s), x\right)\right)} \leq g(s)$ then $\int_{0}^{V(x)} \frac{-d s}{\dot{V}\left(\Phi\left(\theta_{x}(s), x\right)\right)} \leq \int_{0}^{V(x)} g(s) d s<+\infty$ for all $x \in$ $\mathcal{V} \backslash\{0\}$. As $T(0)=0$, we may deduce that the system (1) is finite time stable. Moreover, $\lim _{\|x\| \rightarrow 0} T(x) \leq$ $\lim _{\|x\| \rightarrow 0} \int_{0}^{V(x)} g(t) d t=0$, so the settling-time function is continuous at the origin. To conclude, we may invoke the following result [4, Proposition 2.4.] which shows that $T$ is continuous at the origin if and only if $T$ is continuous on its domain of definition $\mathcal{V}$.

Theorem 2 is quite general. Nevertheless, its application is not easy because the flow is generally unknown. In order to study the stabilization problem, we prefer to restrict the problem to the case of a continuous settling-time function at the origin. We could refer to a result given in [4] in order to use a necessary and sufficient condition involving a Lyapunov function only. We may be inclined to use a more regular settling-time function in order to find a more regular
Lyapunov function. This is important for the problem of stabilization in section IV when using the control Lyapunov functions. For this, we might need the following lemma which is similar to a result given in [5, Lemma 5.1], and [14, Lemma 16].

Lemma 2: Let $\mathcal{O}$ be a non empty open subset of $\mathbb{R}^{n}$, and $\beta: \mathcal{O} \rightarrow \mathbb{R}, \mu, \nu: \mathcal{O} \rightarrow \mathbb{R}_{>0}$ three continuous functions. Suppose $V: \mathcal{O} \rightarrow \mathbb{R}$ is locally Lipschitz on $\mathcal{O}$, if

$$
\forall x \in \mathcal{O},\langle\nabla V(x), f(x)\rangle \leq \beta(x)
$$

then there exists a smooth function $\hat{V}: \mathcal{O} \rightarrow \mathbb{R}$ such that, for all $x \in \mathcal{O}$,

$$
\begin{aligned}
|V(x)-\hat{V}(x)| & \leq \mu(x) \\
\langle\nabla \hat{V}(x), f(x)\rangle & \leq \beta(x)+\nu(x) .
\end{aligned}
$$

Let us recall a result which can be found in $[4$, Theorem 4.2] for the sufficient condition of finite time stability and [4, Theorem 4.3] for the necessary one.

Proposition 1: Consider the system (1) with the uniqueness of solutions in forward time outside the origin, the following properties are equivalent:

1) the origin of the system (1) is finite time stable with a continuous settling-time function at the origin,

2) there exists a real number $c>0, \alpha \in] 0,1[$ and a Lyapunov function $V: \mathcal{V} \rightarrow \mathbb{R}_{\geq 0}$ satisfying

$$
\dot{V}(x) \leq-c(V(x))^{\alpha}
$$

for all $x \in \mathcal{V}$.

The construction of the Lyapunov function, in the proof given in [4, Theorem 4.3], involved the settling-time function in the following sense: $V(x)=T(x)^{\frac{1}{1-\alpha}}$ with $\left.\alpha \in\right] 0,1[$ $\left(\frac{1}{1-\alpha}>1\right)$. As a Lyapunov function is at least continuous, it involves the continuity of the settling-time function at the origin which is equivalent to the continuity of the settlingtime function on its domain of definition (see [4, Proposition 2.4.]).

Now, we may give a variant of this result dedicated to the class $\mathcal{C} \mathcal{L}^{k}$-systems by using the fact that the class of systems with the uniqueness of solutions in forward time is included in the class $\mathcal{C} \mathcal{L}^{k}$-systems for all $k \geq 0$.

Proposition 2: Let $k \geq 0$, if $f$ belongs to the class $\mathcal{C L}^{k}$ then the following properties are equivalent:

1) the origin of the system (1) is finite time stable with a class $\mathcal{C} \mathcal{L}^{0}$ settling-time function,

2) there exists a real number $c>0, \alpha \in] 0,1[$ and a class $\mathcal{C} \mathcal{L}^{\infty}$-Lyapunov function $V: \mathcal{V} \rightarrow \mathbb{R}_{\geq 0}$ satisfying the condition (5).

Moreover, if $V$ is a Lyapunov function satisfying the condition (5) then for all $x \in \mathcal{V}$,

$$
T(x) \leq \frac{V(x)^{1-\alpha}}{c(1-\alpha)} .
$$

Proof: Suppose that 2) is verified. We can find in [4, Theorem 4.2] that the existence of the class $\mathcal{C} \mathcal{L}^{\infty}$-Lyapunov function $V$ satisfying condition (5) implies the finite time stability. Moreover, as $x \mapsto \frac{V(x)^{1-\alpha}}{c(1-\alpha)}$ is a class $\mathcal{C} \mathcal{L}^{\infty}$-function, 
we deduce that $T$ belongs to the class $\mathcal{C} \mathcal{L}^{0}$. Conversely, let us suppose that the origin of the system (1) is finite time stable with a class $\mathcal{C L}^{0}$-settling-time function. By using the proof of proposition 1 which can be found in $[4$, Theorem 4.3], we know that $V(x)=T(x)^{\frac{1}{1-\alpha}}$ with $0<\alpha<1$ belongs to the class $\mathcal{C} \mathcal{L}^{0}(\mathcal{V})$ and is a Lyapunov function for the system (1) satisfying condition (5). Let $0<k<c$, we apply the lemma 2 with the open set $\mathcal{V} \backslash\{0\}$, and $\beta(x)=-c V(x)^{\alpha}, \mu(x)=\frac{1}{2} V(x), \nu(x)=k V(x)^{\alpha}$ to obtain a class $\mathcal{C} \mathcal{L}^{\infty}$-Lyapunov function $\hat{V}$ such that,

$$
\begin{aligned}
\frac{1}{2} V(x) & \leq \hat{V}(x) \leq \frac{3}{2} V(x), \quad x \in \mathcal{V}, \\
\langle\nabla \hat{V}(x), f(x)\rangle & \leq-c^{\prime} \hat{V}(x)^{\alpha}, \quad x \in \mathcal{V} \backslash\{0\},
\end{aligned}
$$

with $c^{\prime}=c-k>0$.

As it is shown in the next two examples, the Lyapunov function satisfying condition (5) may be smooth everywhere.

Example 2 (scalar system): Let $\alpha \in] 0,1[$ and $k>0$, it is easy to see that the basic system

$$
\dot{x}=-k|x|^{\alpha} \operatorname{sgn}(x), \quad x \in \mathbb{R}
$$

is finite time stable using the smooth Lyapunov function $V(x)=\frac{x^{2}}{2}$ with the well known class $\mathcal{C} \mathcal{L}^{\infty}$-settling-time function $T(x)=\frac{|x|^{1-\alpha}}{k(1-\alpha)}$. Indeed, we have for all $x \in \mathbb{R}$

$$
\dot{V}(x)=-k|x|^{1-\alpha}=-2^{\frac{1+\alpha}{2}} k V(x)^{\frac{1+\alpha}{2}}
$$

with $\left.\frac{1+\alpha}{2} \in\right] 0,1[$. For this basic example, the solutions are explicit

$$
\phi^{x_{0}}(t)=\left\{\begin{array}{ll}
g\left(t, x_{0}\right) & \text { if } \quad 0 \leq t \leq \frac{\left|x_{0}\right|^{1-\alpha}}{k(1-\alpha)}, \\
0 & \text { if } t>\frac{\left|x_{0}\right|^{-\alpha}}{k(1-\alpha)}
\end{array},\right.
$$

with $g\left(t, x_{0}\right)=\left(\left|x_{0}\right|^{1-\alpha}-k(1-\alpha) t\right)^{\frac{1}{1-\alpha}} \operatorname{sgn}\left(x_{0}\right)$, so we do not need a Lyapunov function. As it is recalled in the introduction, the settling-time function is given by $T(x)=$ $\int_{x}^{0} \frac{d y}{f(y)}$ for finite time stable scalar systems (see [8] and [12] for a proof of this basic result).

Example 3 (two dimensional system): Let us consider the system:

$$
\left\{\begin{array}{l}
\dot{x}_{1}=-\left|x_{1}\right|^{\alpha} \operatorname{sgn}\left(x_{1}\right)-x_{1}^{3}+x_{2} \\
\dot{x}_{2}=-\left|x_{2}\right|^{\alpha} \operatorname{sgn}\left(x_{2}\right)-x_{2}^{3}-x_{1}
\end{array} .\right.
$$

Taking $V(x)=\frac{\|x\|^{2}}{2}$, we obtain $\dot{V}\left(x_{1}, x_{2}\right)=-\sum_{i=1}^{2}\left(x_{i}^{4}+\right.$ $\left.\left|x_{i}\right|^{\alpha+1}\right) \leq 0 . V$ is a Lyapunov function for the system satisfying $\dot{V}\left(x_{1}, x_{2}\right) \leq-2^{\frac{\alpha+1}{2}} V\left(x_{1}, x_{2}\right)^{\frac{\alpha+1}{2}}$. Indeed, $\sum_{i=1}^{2}\left(x_{i}^{4}+\left|x_{i}\right|^{\alpha+1}\right) \geq\left(x_{1}^{2}+x_{2}^{2}\right)^{\frac{\alpha+1}{2}}=\|x\|^{\alpha+1}$. Thus the origin is finite time stable with a continuous settling-time function verifying $T(x) \leq \frac{2\|x\|^{1-\alpha}}{1-\alpha}$.

\section{Finite time Stabilization of the Class $\mathcal{C} \mathcal{L}^{k}$-AFFINE SYSTEMS}

Let $k \geq 0$, and consider the following affine system

$$
\dot{x}=f_{0}(x)+\sum_{i=1}^{m} f_{i}(x) u_{i}, \quad x \in \mathbb{R}^{n} \text { and } u \in \mathcal{U}
$$

where $\mathcal{U}$ is a non empty open set of $\mathbb{R}^{m}$ containing the origin, $f_{i} \in \mathcal{C} \mathcal{L}^{k}\left(\mathbb{R}^{n}, \mathbb{R}\right)$ for all $0 \leq i \leq m$ and $f_{0}(0)=0$ and the closed-loop system

$$
\dot{x}=f_{0}(x)+\sum_{i=1}^{m} f_{i}(x) u_{i}(x), \quad x \in \mathbb{R}^{n} .
$$

Let us recall the definitions of the stabilization and the finite time stabilization. We will restrict our study to the case of a class $\mathcal{C} \mathcal{L}^{0}$-settling-time function for the finite time stabilization. The control system (6) is stabilizable (respectively finite time stabilizable) if there exists a non empty neighborhood of the origin $\mathcal{V}$ in $\mathbb{R}^{n}$ and a feedback control law $u \in C^{0}(\mathcal{V} \backslash\{0\}, \mathcal{U})$ such that:

1) $u(0)=0$,

2) the origin of the system (7) is asymptotically stable (respectively finite time stable with a class $\mathcal{C} \mathcal{L}^{0}$-settlingtime function).

Here, we give a necessary and sufficient condition for the finite time stabilization of the system (6) involving the continuity of the settling-time function at the origin for the closed-loop system (7). We add a condition to the concept of control Lyapunov function first defined in [2], which leads to the finite time stabilization.

We are going to recall some usual definitions. A positive definite function $V \in \mathcal{C L}^{\infty}\left(\mathcal{V}, \mathbb{R}_{\geq 0}\right)$ is a control Lyapunov function for the system (6) if for all $x \in \mathcal{V} \backslash\{0\}$,

$$
\inf _{u \in \mathcal{U}}(a(x)+\langle B(x), u\rangle)<0 .
$$

where $a(x)=\mathcal{L}_{f_{0}} V(x), B(x)=\left(b_{1}(x), \ldots, b_{m}(x)\right)$ with $b_{i}(x)=\mathcal{L}_{f_{i}} V(x)$ for $1 \leq i \leq m$.

To obtain the finite time stabilization, we have to bring in the control Lyapunov function $V$ the following condition which holds for all $x \in \mathcal{V} \backslash\{0\}$ and for a real number $\alpha \in] 0,1[$

$$
\inf _{u \in \mathcal{U}}(a(x)+\langle B(x), u\rangle) \leq-c(V(x))^{\alpha} .
$$

As usual, such a control Lyapunov function satisfies the small control property if for each $\epsilon>0$, there exists $\delta>0$ such that, if $x \in \delta \mathcal{B}^{n}$, then there exists some $u \in \epsilon \mathcal{B}^{m}$ such that

$$
a(x)+\langle B(x), u\rangle<0 .
$$

Remark 3: If $\mathcal{U} \subset \mathbb{R}$, the small control property is equivalent to

$$
\limsup _{\|x\|_{n} \rightarrow 0} \frac{a(x)}{|B(x)|} \leq 0 .
$$

The limit may very well be $-\infty$.

We set $b(x)=\|B(x)\|^{2}$. The theorem 3 of Mickael will help us to show our main result on finite time stabilization. 
Theorem 3 (of Mickael): Let $\mathcal{X}$ and $\mathcal{Y}$ be two metric spaces, for every lower semi-continuous (respectively locally Lipschitz) set-valued function $\Phi: \mathcal{X} \rightarrow 2^{\mathcal{Y}}, x \mapsto \Phi(x)$ where $2^{\mathcal{Y}}$ will denote the family of non-empty, closed, convex subsets of $\mathcal{Y}$ it is possible to extract a continuous (respectively locally Lipschitz) function $f$ such that $f(x) \in$ $\Phi(x)$ for all $x \in \mathcal{X}$.

Theorem 4: The system (6) is finite time stabilizable under a class $\mathcal{C L}^{0}$-feedback control if and only if there exists a control Lyapunov function for the system (6) which satisfies the condition (8) for a real number $\alpha \in] 0,1[$ and the small control property.

Proof: If the control system (6) is finite time stabilizable, then the closed-loop system (7) is finite time stable with a class $\mathcal{C} \mathcal{L}^{0}$-settling-time function. By using proposition 2, there exists a class $\mathcal{C} \mathcal{L}^{\infty}$-Lyapunov function $V$ for the closed-loop system (7) satisfying the condition (5) which implies that (8) is valid. Moreover, by using the feedback control $u(x)$ and its continuity, it is easy to see that the control Lyapunov function satisfies the small control property. Conversely, if there exists a control Lyapunov function $V: \mathcal{V} \rightarrow \mathbb{R}_{\geq 0}$ for the system (6) satisfying condition (8), then we introduce the set valued function $\Phi$ defined for $x \in \mathcal{V} \backslash\{0\}$ by

$$
\Phi(x)=\left\{v \in \mathcal{U}: a(x)+\langle B(x), v\rangle \leq-c(V(x))^{\alpha}\right\} .
$$

As $v \mapsto a(x)+\langle B(x), v\rangle$ is affine, it implies that for all $x \in \mathcal{V} \backslash\{0\}, \Phi(x)$ belongs to the family of non-empty closed convex subsets of $\mathcal{U}$ for the subspace topology. As $f_{i}$ belongs to the class $\mathcal{C} \mathcal{L}^{k}$ for all $0 \leq i \leq m$ and $V \in \mathcal{C} \mathcal{L}^{\infty}\left(\mathcal{V}, \mathbb{R}_{\geq 0}\right)$, $a(x)+\langle B(x), v\rangle+c(V(x))^{\alpha}$ is locally Lipschitz for all $x \in \mathcal{V} \backslash\{0\}$. Thus, we may deduce that $\Phi$ is locally Lipschitz on $\mathcal{V} \backslash\{0\}$. As $V$ satisfies the small control property, it is shown in [2, Theorem 4.3] that we may extend $\Phi$ on $\mathcal{V}$ by $\Phi(0)=\{0\}$ such that $\Phi$ now is lower semi-continuous on $\mathcal{V}$. We may apply the theorem 3 of Mickael to find a selection $u \in \mathcal{C L}^{0}(\mathcal{V}, \mathcal{U})$. Then $V$ is a class $\mathcal{C} \mathcal{L}^{\infty}$-Lyapunov function for the closed loop system (7) satisfying the condition 5. Thus, by using proposition 2 we deduce that the system (6) is finite time stabilizable.

Theorem 4 provides a tool for the finite time stabilization with a class $\mathcal{C} \mathcal{L}^{0}$-settling-time function.

In practical terms, the resolution of the finite time stabilization is a delicate task which has generally been studied for homogeneous systems of negative degree with respect to a flow of a complete vector field. Indeed, for this kind of systems, finite time stability is equivalent to asymptotic stability (see [3], [9] for more details). Nevertheless, if we want to use a control Lyapunov function to obtain a constructive feedback control for finite time stabilization, we can use a modified version of the Sontag feedback control given in [13].

Lemma 3: If there exists a continuously differentiable control Lyapunov function $V: \mathcal{V} \rightarrow \mathbb{R}_{\geq 0}$ for the control system (6), then it is stabilizable under the feedback control

$$
\begin{aligned}
& u(x)=\left(u_{1}(x), \ldots, u_{m}(x)\right) \text { defined by } \\
& u_{i}(x)=\left\{\begin{array}{lll}
-b_{i}(x) \frac{a(x)+\sqrt[p]{a(x)^{p}+b(x)^{q}}}{b(x)} & \text { if } & x \in \mathcal{V} \backslash\{0\} \\
0 & \text { if } & x=0
\end{array}\right.
\end{aligned}
$$

where $p, q \geq 2$ are even integers. If furthermore $V$ satisfies the small control property, then the feedback control (9) is also continuous at the origin.

Proof: Suppose there exists a smooth control Lyapunov function $V: \mathcal{V} \rightarrow \mathbb{R}_{\geq 0}$. Let

$$
E=\left\{(x, y) \in \mathbb{R}^{2}: x<0 \text { or } y>0\right\}
$$

and $\varphi$ a function defined on $E$ by

$$
\varphi(x, y)=\left\{\begin{array}{lll}
\frac{x+\sqrt[p]{x^{p}+y^{q}}}{y} & \text { if } & y \neq 0 \\
0 & \text { if } & y=0
\end{array} .\right.
$$

As

$$
\begin{aligned}
\lim _{y \rightarrow 0} \frac{x+\sqrt[p]{x^{p}+y^{q}}}{y} & =\lim _{y \rightarrow 0} \frac{x+|x| \sqrt[p]{1+\frac{y^{q}}{x^{p}}}}{y} \\
& =\lim _{y \rightarrow 0} \frac{-y^{q-1}}{p x^{p-1}}=0,
\end{aligned}
$$

$\varphi$ is continuous on $E$. As $V$ is a control Lyapunov function, then we know that $(a(x), b(x)) \in E$ for all $x \in \mathcal{V} \backslash\{0\}$. Thus, we define the feedback control by $u_{i}(x)=-b_{i}(x) \varphi(a(x), b(x)) . u(x)$ is continuous on $\mathcal{V} \backslash\{0\}$ and we obtain for all $x \in \mathcal{V} \backslash\{0\}$

$$
\begin{aligned}
\left\langle\nabla V(x), f_{0}(x)+\sum_{i=1}^{m} f_{i}(x) u_{i}(x)\right\rangle & =-\sqrt[p]{a(x)^{p}+b(x)^{q}} \\
& <0 .
\end{aligned}
$$

So, $V$ is a Lyapunov function for the closed-loop system (7), and by using the Lyapunov theorem we know that the origin of the closed loop system (7) is asymptotically stable. The proof concerning the stabilization under the small control property is similar to the one given in [13, Theorem 1].

Proposition 3: If there exists a continuously differentiable control Lyapunov function $V: \mathcal{V} \rightarrow \mathbb{R}_{\geq 0}$ for the control system (6) verifying the small control property and for all $x \in \mathcal{V}$,

$$
\sqrt[p]{a(x)^{p}+b(x)^{q}} \geq c V(x)^{\alpha}
$$

where $p, q \geq 2$ are even integers, and where $c>0$ and $0<\alpha<1$ then the system (6) is finite time stabilizable under the continuous feedback control (9).

Proof: The asymptotic stability is proved using Lemma 3. It is shown in [13, Theorem 1] that if $V$ satisfies the small control property, the feedback control (9) is continuous at the origin. The inequality

$$
\begin{aligned}
\left\langle\nabla V(x), f_{0}(x)+\sum_{i=1}^{m} f_{i}(x) u_{i}(x)\right\rangle & =-\sqrt[p]{a(x)^{p}+b(x)^{q}} \\
& \leq-c V(x)^{\alpha}
\end{aligned}
$$

ensures the finite time convergence by using proposition 1 . 
Remark 4: Proposition 3 is also true for an only continuous control system (6). The proof of the finite time convergence can be given by using a result of Haimo in [8, Proposition 1].

Example 4: Let $0<\beta, \gamma<1$ such that $4(\beta+\gamma)<\beta+1$ (for example $\beta=\gamma=\frac{1}{8}$ ) and consider the system

$$
\left\{\begin{array}{l}
\dot{x}_{1}=-\left|x_{1}\right|^{\gamma} \operatorname{sgn}\left(x_{1}\right)-x_{2} \\
\dot{x}_{2}=\left|x_{1}\right|^{\beta} \operatorname{sgn}\left(x_{1}\right)\left|x_{2}\right|^{1-\beta}+\left|x_{2}\right|^{\gamma} u
\end{array} .\right.
$$

Using the class $C^{1}$-function $V(x)=\left|x_{1}\right|^{\beta+1}+\left|x_{2}\right|^{\beta+1}$, we obtain

$$
\begin{aligned}
a(x) & =-(\beta+1)\left|x_{1}\right|^{\beta+\gamma} \\
B(x) & =(\beta+1)\left|x_{2}\right|^{\beta+\gamma} \operatorname{sgn}\left(x_{2}\right) \\
b(x) & =(\beta+1)^{2}\left|x_{2}\right|^{2(\beta+\gamma)} .
\end{aligned}
$$

As $\inf _{u \in \mathbb{R}}(a(x)+B(x) u)<0$ for $x \neq 0, V$ is a control Lyapunov function for the system. The fact that

$$
\frac{a(x)}{|B(x)|}=\frac{-\left|x_{1}\right|^{\beta+\gamma}}{\left|x_{2}\right|^{\beta+\gamma}} \leq 0
$$

and remark 3 implies that $V$ satisfies the small control property. Now, by using the feedback control (9) with $(p, q)=(4,2)$, we obtain

$$
\begin{aligned}
a(x)^{4}+b(x)^{2} & =(\beta+1)^{4}\left(\left|x_{1}\right|^{4(\beta+\gamma)}+\left|x_{2}\right|^{4(\beta+\gamma)}\right) \\
& \geq(\beta+1)^{4}\left(\left|x_{1}\right|^{\beta+1}+\left|x_{2}\right|^{\beta+1}\right)^{\frac{4(\beta+\gamma)}{\beta+1}}, \\
& \geq(\beta+1)^{4} V(x)^{\frac{4(\beta+\gamma)}{\beta+1}} .
\end{aligned}
$$

with $0<\frac{4(\beta+\gamma)}{\beta+1}<1$. Thus $\sqrt[4]{a(x)^{4}+b(x)^{2}} \geq$ $(\beta+1) V(x)^{\alpha}$ with $\alpha=\frac{\beta+\gamma}{\beta+1}<1$, by using proposition (3) we know that the control system is finite time stabilizable under the continuous feedback control

$$
u(x)=\frac{\left|x_{1}\right|^{\beta+\gamma}-\sqrt[4]{\left|x_{1}\right|^{4(\beta+\gamma)}+\left|x_{2}\right|^{4(\beta+\gamma)}}}{\left|x_{2}\right|^{\beta+\gamma} \operatorname{sgn}\left(x_{2}\right)} .
$$

\section{CONCLUDING REMARKS}

The problem of finite time stability of systems with the uniqueness of solutions in forward time is solved for differential equations by giving a necessary and sufficient condition for the finite time stability involving a Lyapunov function. Our results bring an answer to the question asked by Bhat and Bernstein in the conclusion of their paper [4] concerning a stronger converse result for finite time stability. Moreover, by using their results on finite time stability involving continuity of the settling-time function at the origin, we have succeeded to solve the problem of the finite time stabilization of class $\mathcal{C} \mathcal{L}^{k}$-affine systems involving a class $\mathcal{C} \mathcal{L}^{0}$-settling-time function. The universal controller given by Sontag in [13] is extended to design a feedback control for the finite time stabilization. Nevertheless, our paper raises certain questions that are important from the point of view of the stabilization theory, in particular the construction of a universal finite time feedback control using a control Lyapunov function satisfying condition (8).

\section{REFERENCES}

[1] R. P. Agarwal and V. Lakshmikantham. Uniqueness and Nonuniqueness Criteria for Ordinary Differential Equations. Ser. Real Anal. 6, World Scientic, Singapore, 1993.

[2] Z. Artstein. Stabilization with relaxed controls. Nonlinear Anal., 7(11):1163-1173, 1983.

[3] S. P. Bhat and D. Bernstein. Continuous, bounded, finite-time stabilization of the translational and rotational double integrator. In IEEE Conference on Control Applications, pages 185-190, Dearborn, MI, 1996.

[4] S. P. Bhat and D. S. Bernstein. Finite time stability of continuous autonomous systems. SIAM J. Control Optim., 38(3):751-766, 2000.

[5] F. H. Clarke, Yu S. Ledyaev, and R. J. Stern. Asymptotic stability and smooth Lyapunov function. J. Differential Equations, 149:69-114, 1998.

[6] A. F. Filippov. Differential Equations with Discontinuous Righthand Sides. Kluwer Academic Publishers, Dordrecht/Boston/London, 1988.

[7] W. Hahn. Theory and Application of Liapunov's Direct Method. Prentice-Hall inc., 1963. N.J.

[8] V. T. Haimo. Finite time controllers. SIAM J. Control Optim., 24(4):760-770, 1986.

[9] Y. Hong. Finite-time stabilization and stabilizability of a class of controllable systems. Systems Control Lett., 46:231-236, 2002.

[10] M. Kawski. Stabilization of nonlinear systems in the plane. Systems Control Lett., 12:169-175, 1989.

[11] J. Kurzweil. On the inversion of Liapunov's second theorem on stability of motion. Amer. Math. Soc. Transl., 24:19-77, 1963.

[12] E. Moulay and W. Perruquetti. Finite time stability of non linear systems. In IEEE Conference on Decision and Control, pages 36413646, Hawaii, USA, 2003.

[13] E. Sontag. A universal construction of Arststein's theorem on nonlinear stabilization. Systems Control Lett., 13:117-123, 1989.

[14] A. R. Teel and L. Praly. A smooth Lyapunov function from a class-KL estimate involving two positive semidefinite functions. ESAIM Control Optim. Calc. Var., 5:313-367, 2000. 